\title{
Collagen synthesis disruption and downregulation of core elements of TGF- $\beta$, Hippo, and Wnt pathways in keratoconus corneas
}

\begin{abstract}
Michal Kabza ${ }^{1,2,6}$, Justyna A Karolak ${ }^{1,3,6}$, Malgorzata Rydzanicz ${ }^{4}$, Michał W Szcześniak², Dorota M Nowak ${ }^{1,3}$, Barbara Ginter-Matuszewska ${ }^{1,3}$, Piotr Polakowski ${ }^{5}$, Rafal Ploski ${ }^{4}$, Jacek P Szaflik ${ }^{5}$ and Marzena Gajecka ${ }^{\star, 1,3}$

To understand better the factors contributing to keratoconus (KTCN), we performed comprehensive transcriptome profiling of human KTCN corneas for the first time using an RNA-Seq approach. Twenty-five KTCN and 25 non-KTCN corneas were enrolled in this study. After RNA extraction, total RNA libraries were prepared and sequenced. The discovery RNA-Seq analysis (in eight KTCN and eight non-KTCN corneas) was conducted first, after which the replication RNA-Seq experiment was performed on a second set of samples (17 KTCN and 17 non-KTCN corneas). Over $82 \%$ of the genes and almost $75 \%$ of the transcripts detected as differentially expressed in KTCN and non-KTCN corneas were confirmed in the replication study using another set of samples. We used these differentially expressed genes to generate a network of KTCN-deregulated genes. We found an extensive disruption of collagen synthesis and maturation pathways, as well as downregulation of the core elements of the TGF- $\beta$, Hippo, and Wnt signaling pathways influencing corneal organization. This first comprehensive transcriptome profiling of human KTCN corneas points further to a complex etiology of KTCN.
\end{abstract}

European Journal of Human Genetics (2017) 25, 582-590; doi:10.1038/ejhg.2017.4; published online 1 February 2017

\section{INTRODUCTION}

Keratoconus (KTCN, OMIM 148300) is a degenerative eye disorder characterized by progressive stromal thinning, resulting in a conical shape of the cornea. Structural changes in the corneal layers cause optical aberrations, leading to a loss of visual function. ${ }^{1}$ The estimated frequency of KTCN in the general population is 1 in 2000 individuals. ${ }^{1}$ Corneal transplant surgery becomes necessary in advanced KTCN, because of a progression of stromal thinning or scarring. ${ }^{2}$

KTCN development may be related to environmental factors such as constant eye rubbing or contact lens wear, or it may be genetically controlled. ${ }^{3}$ Recently, it was suggested that KTCN could be a quasiinflammatory condition. ${ }^{4}$ However, the exact causes of this disease are not well characterized, and its complexity and heterogeneity make it difficult to discover the factors that unambiguously trigger the KTCN phenotype.

Over the years, numerous functional and positional candidate genes for KTCN have been proposed, including VSX1, HGF, and mir-184..$^{5-7}$ Also, numerous sequence variants in our KTCN research were identified. ${ }^{8-12}$ However, because variations in these genes were specific to particular families or populations only, they could not be recognized as causative for KTCN in the general population. The one possible explanation for the absence of genetic KTCN marker(s) is that the studied genes constitute only a minor fraction of the total genetic load and the number of genes altered in KTCN may be higher. It is also possible that the KTCN phenotype is not linked to variations in the nucleotide sequence. Thus, it has been assumed that in addition to DNA-based analysis, RNA studies may provide important insights into KTCN etiology. 13,14

Herein, we present the results of comprehensive transcriptome profiling of KTCN corneas and non-KTCN corneas, obtained for the first time using an RNA-Seq approach. Distinct differences in gene expression patterns between the KTCN and non-KTCN corneas were found. The categorization of the upregulated and downregulated genes into functionally relevant groups identified molecular pathways deregulated in KTCN corneas - the TGF- $\beta$, Hippo, and Wnt pathways - and allowed a better understanding of the molecular bases of this disease.

\section{MATERIALS AND METHODS}

Patients and clinical evaluation

All study participants were recruited and examined in the Department of Ophthalmology, Medical University of Warsaw, Warsaw, Poland. The clinical characteristics of all individuals are shown in Supplementary Table 1. The ophthalmic examination consisted of a best-corrected visual acuity evaluation, intraocular pressure assessment, slit-lamp examination, and corneal topography and pachymetry assessments. Patients were considered affected if they had at least one clinical sign - corneal thinning, Vogt's striae, or Fleischer rings - in slit-lamp biomicroscopy concurrent with the characteristic deviation from normal parameters based on the topography and pachymetry assessments. The study protocol was approved by the Institutional Review Board at Poznan University of Medical Sciences, Poznan, Poland. The possible consequences of the study were explained, and informed consent was obtained from all participants before participation, according to the Declaration of Helsinki.

${ }^{1}$ Department of Genetics and Pharmaceutical Microbiology, Poznan University of Medical Sciences, Poznan, Poland; ${ }^{2}$ Department of Bioinformatics, Institute of Molecular Biology and Biotechnology, Adam Mickiewicz University in Poznan, Poznan, Poland; ${ }^{3}$ Institute of Human Genetics, Polish Academy of Sciences, Poznan, Poland; ${ }^{4}$ Department of Medical Genetics, Medical University of Warsaw, Warsaw, Poland; ${ }^{5}$ Department of Ophthalmology, Medical University of Warsaw, Warsaw, Poland

*Correspondence: Dr M Gajecka, Department of Genetics and Pharmaceutical Microbiology, Poznan University of Medical Sciences, Swiecickiego 4, Poznan 60-781, Poland. Tel: +4861 854 6721; Fax: +4861 854 6720; E-mail: gamar@man.poznan.pl

6 These authors contributed equally to this work.

Received 22 June 2016; revised 16 November 2016; accepted 24 December 2016; published online 1 February 2017 


\section{Study subjects and material designation}

The $25 \mathrm{KTCN}$ corneal tissues were derived from non-related Polish patients during a keratoplasty procedure for KTCN. The 25 non-KTCN corneas, used as controls, were collected from patients who were referred for corneal transplantation for different reasons, such as bullous keratopathy, corneal scarring, ulcers, and perforations. The corneas were submersed in an RNA stabilization solution (RNAlater; Qiagen, Hilden, Germany) directly after corneal button excision during the surgical procedure, and stored at $-20^{\circ} \mathrm{C}$ until RNA extraction.

\section{RNA extraction}

The whole corneal buttons were transferred to lysis solution (Norgen Biotek, Thorold, ON, Canada) in a microtube with Lysing Matrix D (MP Biomedicals, Irvine, CA, USA) and homogenized using a FastPrep-24 Instrument (MP Biomedicals). Further total RNA extraction and purification steps were performed according to the instructions supplied with a Total RNA Purification Kit (Norgen Biotek). Next, an on-column DNase I (Zymo Research, Irvine, CA, USA) treatment was performed. The quality and quantity of the purified RNA samples were assessed using an RNA 6000 Nano Kit (Agilent Technologies, Waldbronn, Germany). The concentrated samples of total RNA were stored frozen at $-80^{\circ} \mathrm{C}$ until further analysis.

\section{Total RNA library preparation and sequencing}

Libraries were prepared with a TruSeq Stranded Total RNA LT with Ribo-Zero Human/Mouse/Rat Kit (Illumina, San Diego, CA, USA) according to the manufacturer's protocol, with slight modifications. Briefly, total RNA samples were depleted of both cytoplasmic and mitochondrial rRNA using biotinylated, target-specific oligos combined with Ribo-Zero rRNA removal beads. Then, the total RNA samples were fragmented to generate inserts ranging in size from 120 to $200 \mathrm{bp}$ (median size, $150 \mathrm{bp}$ ); fragmentation time was adjusted to sample RNA integrity number (RIN) to avoid RNA over-fragmentation. SuperScript II Reverse Transcriptase (Invitrogen, Carlsbad, CA, USA) and random hexamer primers were used to synthesize the first-strand CDNA, and DNA polymerase I and RNase $\mathrm{H}$ were used to synthesize the second-strand cDNA. Doublestranded cDNA samples were subjected to classical library preparation steps, including end repair, $3^{\prime}$ end adenylation, indexed adapter ligation, and 12 cycles of PCR enrichment amplification. The generated libraries were assessed using an Agilent DNA 1000 Kit (Agilent Technologies) and quantified using a Qubit dsDNA High Sensitivity Kit (Life Technologies, Eugene, OR, USA). Finally, cluster generation was performed using a TruSeq PE Cluster Kit v3 or v4 (Illumina) on a cBot instrument (Illumina). A 75- or 100-bp paired-end run was performed on a HiSeq1500 platform (Illumina). RIN scores, RNA input, RNA fragmentation time, and sequencing conditions are summarized in Supplementary Table 2 .

The cornea samples were assessed in two independent RNA-Seq experiments. The first case-control study (the discovery study) was performed in the direction of novel transcript discovery and differential expression analysis. The tested materials were eight KTCN corneas and eight non-KTCN corneas sequenced at a depth of 150-250 million read pairs per sample. The second case-control study (the replication study) was used for replicating the differential expression analysis. The tested materials were $17 \mathrm{KTCN}$ and 17 non-KTCN samples sequenced with coverage of 50-70 million read pairs per sample.

\section{RNA-Seq data analysis}

Short reads obtained during sequencing were processed using Trimmomatic ${ }^{15}$ to remove adapter sequences and poor quality regions. Reads that were similar to human rRNA sequences were removed using a Bowtie 2 short read mapper. ${ }^{16}$ Filtered reads from the discovery study were mapped to the reference human genome (GRCh38) using the STAR 2-pass mapping approach. ${ }^{17}$

Transcripts were assembled from mapped reads using StringTie software, ${ }^{18}$ requiring minimum coverage of 10 reads for assembled transcripts and detected splicing junctions. Assembled transcripts shorter than $200 \mathrm{bp}$ were discarded. The mitochondrial genome was excluded from the transcript assembly as suggested in the StringTie documentation. ${ }^{18}$
Assembled transcripts were merged with reference GENCODE 21 annotations using Cuffmerge ${ }^{19}$ to detect novel genes and isoforms. Novel transcripts were only kept if they contained at least one intron. The protein-coding potential of the transcripts was predicted using TransDecoder. ${ }^{20}$

The expression of analyzed genes and transcripts, both reference and novel, was estimated with Kallisto. ${ }^{21}$ Differential expression analyses were performed separately for the discovery and replication data sets using DESeq2 and edgeR packages. ${ }^{22,23}$ Only genes and transcripts with at least 10 reads assigned to them in each compared sample were tested for differential expression, in accordance with the bioinformatics analysis guide by Cofactor Genomics (https:// cofactorgenomics.com/6-changes-thatll-make-big-difference-rna-seq-part-6/). Genes and transcripts were considered to be differentially expressed if they had a $<0.01$ false discovery rate and a $>1.5$-fold change. Diagnostic charts, such as heat maps and PCA and MA plots, were created using functionality provided by the DESeq2 package.

\section{Pathway overrepresentation analysis}

The overrepresentation analysis of molecular pathways among genes upregulated and downregulated in KTCN was performed using the ConsensusPathDB server. ${ }^{24}$ Only genes that were consistently classified as differentially expressed by both DESeq 2 and edgeR in the discovery and replication studies were used for overrepresentation analysis. Genes expressed in the cornea with a mean expression level of at least 1 transcripts per million (TPM) were used as the background gene set. We were only interested in pathways sharing at least five genes with our gene sets, and we used a q-value cutoff of 0.01 .

\section{Reverse transcription-quantitative polymerase chain reaction} (RT-qPCR)

RNA-Seq data for selected genes, including TGFB3, CTGF, and COL21A1, were verified using the LightCycler 96 system (Roche Diagnostics, Penzberg, Germany) and the RealTime ready Custom Panel 96 (Roche), according to the manufacturer's protocol. The expression levels of our targets of interest in six KTCN and six non-KTCN corneas were quantify by GenEx Enterprise version 6 software (MultiD Analyses AB, Gothenburg, Sweden).

\section{RESULTS}

\section{RNA-Seq quality control and assessment}

The discovery RNA-Seq analysis (in eight KTCN and eight non-KTCN corneas) was conducted first, after which the replication RNA-Seq experiment was performed on a second set of samples (17 KTCN and 17 non-KTCN corneas). Short reads obtained during the discovery and replication studies were subjected to quality control and removal of rRNA sequences. In all cases, $>80 \%$ of the read pairs passed the quality filtering, and in the vast majority of samples, the fraction of rRNAs did not exceed 5\%. Short reads from the discovery study were mapped to the reference human genome, with only $1-2 \%$ failing to map. Quality filtering and mapping statistics for the discovery and replication analyses are presented in Supplementary Table 3.

\section{Detection of novel genes and isoforms}

Overall, 59795 novel transcripts and 108 previously unannotated genes were found. The protein-coding potential of the novel transcripts was predicted, and different classes of detected novel transcripts ('class codes' in Cufflinks terminology) turned out to have various protein-coding preferences. The novel intergenic loci contained a much greater proportion of noncoding RNAs than the novel isoforms of the known genes (Supplementary Table 4). The final merged annotations obtained in this study are provided in Supplementary File 1. The expression values of genes and transcripts are provided in Supplementary File 2. 


\section{Differential expression analysis}

Sample clustering and principal component analysis (PCA) based on detected differentially expressed genes showed that three of eight nonKTCN samples in the discovery study (KR_53, KR_57, KR_64) and two of 17 non-KTCN samples in the replication study (KR_08, KR_35) clustered with the KTCN samples, not with other non-KTCN samples (Supplementary File 3). This pattern may have been the result of high heterogeneity among the non-KTCN samples; the samples were excluded from further analysis, as recommended in the RNA-Seq analysis protocol of the EpiGeneSys project (http://www.epigenesys.eu/ images/stories/protocols/pdf/20150303161357_p67.pdf). Removing the samples resulted in a distinct separation between non-KTCN and KTCN samples, as shown in the heat maps and PCA plots provided (Figure 1). All diagnostic plots for the final differential expression analysis are presented in Supplementary File 4.

In the discovery study, 2650 genes exhibiting differential expression were identified, of which 795 (30\%) were upregulated and 1855 (70\%) were downregulated. In addition, $1937(73.09 \%)$ of the genes had coverage ( $\geq 10$ reads) sufficient to be tested in the discovery and replication studies; 1599 (82.55\%) genes were recognized by both packages (DESeq2 and edgeR), 33 (1.70\%) were recognized by only one package, and $305(15.75 \%)$ were not observed in the replication study. Similarly, 5235 differentially expressed transcripts (1578 upregulated and 3657 downregulated) were found in the discovery analysis, $2744(52.42 \%)$ of which were also tested during replication. Of those, 2057 (74.96\%) were recognized by both packages, 56 (2.04\%) were recognized by only one package, and 631 (23\%) were not found in the replication study. Detailed results of the differential expression analyses of both gene and transcript levels are presented in Supplementary File 5.

\section{Validation of the RNA-Seq differential expression analysis by RT-qPCR}

Quantitative real-time PCR analysis of selected genes in KTCN and non-KTCN individuals showed a 2.13-fold and 2.88-fold lower a
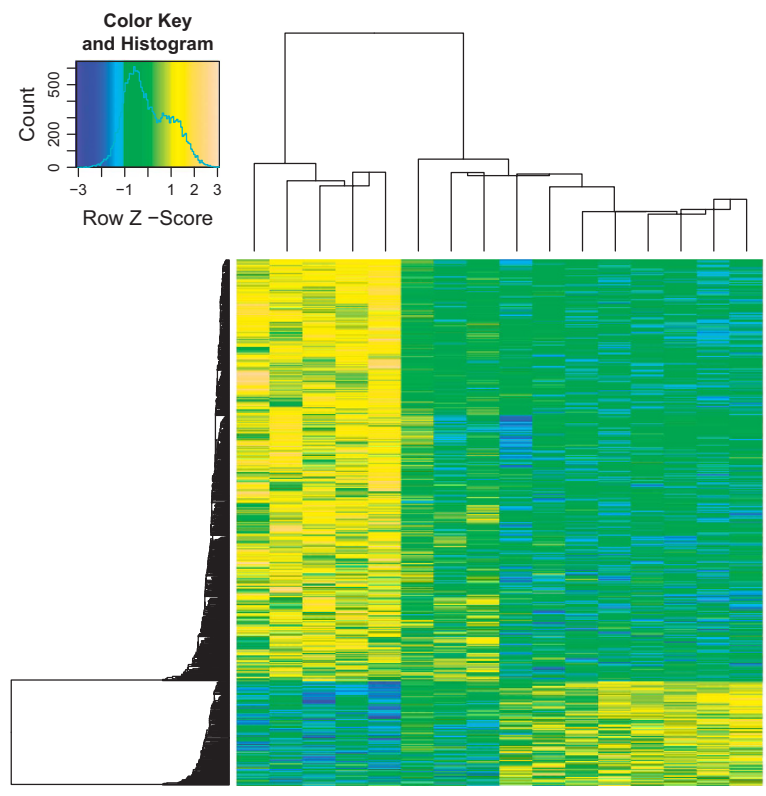

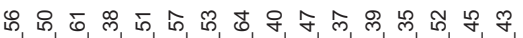

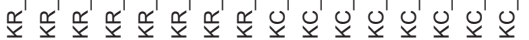

b

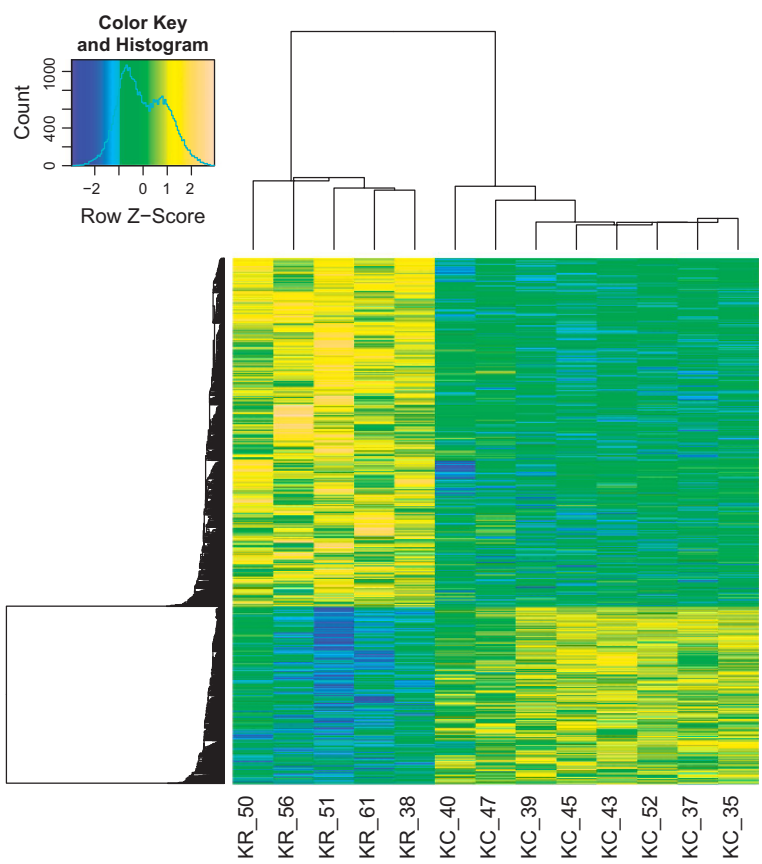

d

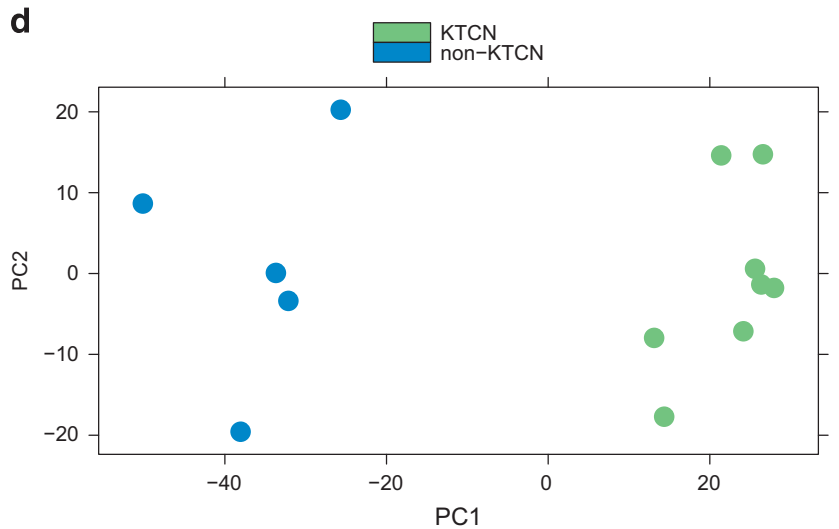

Figure 1 Hierarchical clustering of KTCN and non-KTCN samples and PCA plots in the discovery study. Heat maps indicate hierarchical clustering of KTCN and non-KTCN samples based on the expression values of differentially expressed genes before removing the samples (a) and after removing the samples (b). PCA plots are based on the expression values of all genes before removing the samples (c) and after removing the samples (d). 
expression of TGFB3 and CTGF genes, respectively, and 2.47-fold higher expression of COL21A1 gene in KTCN patients (Figure 2).

Pathway overrepresentation analysis across differentially expressed genes

Overrepresentation analyses of molecular pathways were performed separately on the upregulated and downregulated genes. The top overrepresented pathways detected in both data sets are presented in Table 1. In the upregulated gene set, only a few pathways showed significant KTCN overrepresentation, including 'CREB phosphorylation through the activation of Ras' and 'Post NMDA receptor activation events.'

Downregulated genes were enriched in 51 different pathways, including pathways related to collagen formation and modification, as well as extracellular matrix (ECM) organization and focal adhesion (Table 2). Overrepresentation of the broad range of cytokine ligands and receptors downregulated in KTCN corneas was also observed. The only cytokine-related pathway affected as a whole seemed to be the Hippo signaling pathway annotated in the KEGG database. However, it should be considered a Hippo pathway sensu lato, as it encompasses core elements of the TGF- $\beta$, Hippo, and Wnt pathways. ${ }^{25}$ Vital elements of these three pathways are downregulated in $\mathrm{KTCN}$, including TGF- $\beta$ ligands (TGFB1, TGFB3) and receptors (TGFBR1, TGFBR2), a Wnt ligand (WNT5A) and receptors (FZD1, FZD7), regulatory proteins (AXIN2, SMAD7, ID2), kinases (LATS2, $P P P 2 R 2 B$ ), and transcription factors (TEAD2, TEAD4; Figure 3). Connective tissue growth factor $(C T G F)$ is an example of a gene controlled by the three aforementioned pathways; with a 10.33 -fold change, it represents one of the most strongly downregulated genes. The detailed results of the pathway overrepresentation analysis are presented in Supplementary File 6.

\section{DISCUSSION}

In this study, RNA-Seq was used for comprehensive transcriptome analysis of human corneas derived from living donors referred for corneal transplantation. Some previous RNA studies of KTCN were conducted using corneal cell lines or tear samples, neither of which reflects exactly the properties of in vivo corneal cells. To achieve sufficient statistical power to identify differentially expressed genes, ${ }^{26}$ 50 RNA samples derived from the corneas of $25 \mathrm{KTCN}$ patients and

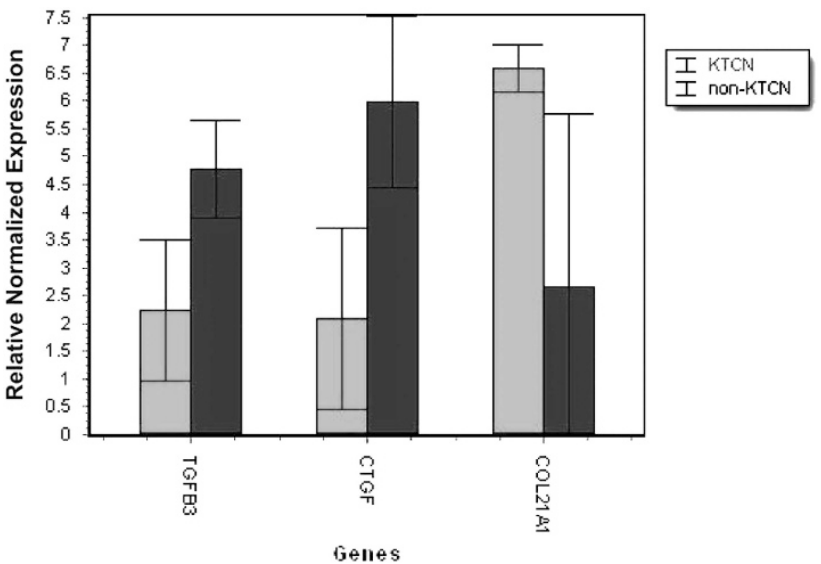

Figure 2 Relative quantification and comparison of the expression of TGFB3, CTGF, and COL21A1 genes in KTCN and non-KTCN individuals. Bars indicate mean expression (white in KTCN, black in non-KTCN); the error bar show SD, $P$-value $<0.01$.
25 non-KTCN individuals were tested. The relatively large sample size and the RNA-Seq approach enhanced with modern bioinformatics analysis enabled the most comprehensive evaluation of transcriptomes in human KTCN subjects to date.

The first transcriptome-level details of KTCN samples were reported in 2004, based on a microarray study of human cultured keratocytes derived from five KTCN corneas. The significant differential expression of TNFAIP6, IGFBP5, and IGFBP3 out of 164 analyzed genes, was identified. ${ }^{27}$ In another study, a high-density oligonucleotide microarray containing probes corresponding to 5600 genes revealed the abnormal expression of 56 genes in KTCN corneal epithelium samples compared with normal corneal epithelial cells. ${ }^{13,28}$ Of those, ACTN1, SERPINB1, LOX, SLPI, DSG3, FHL2, ANXA1, LGALS1, RPL23, S100A2, and GPNMB were also expressed differentially in our study. In the cited report, all 11 genes were upregulated, while in the patients examined by RNA-Seq in this study, these genes were downregulated. More recently, Macé et al ${ }^{14}$ reported transcriptome results from a genome-wide microarray from $10 \mathrm{KTCN}$ corneas and 10 control corneal tissues from unrelated patients who underwent enucleation for choroidal melanomas. Eighty-seven genes showed significant differential expression, including the mucins, keratins, and genes involved in fibroblast proliferation. In the current investigation, only results regarding $A C T B, C D K N 1 A, M C L 1$, and JUN genes were replicated as downregulated genes. The latest study performed by RNA-Seq in induced pluripotent stem cells (iPSC) derived from KTCN fibroblasts and iPSC derived from normal corneal fibroblasts revealed different expression of COL5A1, FGFR2, MMP9, TGFBR3, COL4A1, COL4A2, IL6, and HGF. ${ }^{29}$ In our study, none of these genes were differentially expressed. Taken together, the previous studies have identified a number of abnormally expressed genes in KTCN corneas, but only a small proportion of them were replicated in further studies. Dissimilarities among the results may be due to the use of different types of tested cells, limited numbers of samples, different techniques, or various array probe sets used in the various studies.

Here, a high coverage RNA sequencing of 150-250 million read pairs per sample, was performed in the discovery study. Although this sequencing depth significantly exceeded the depth necessary to estimate gene expression levels, it enabled the detection of novel transcripts in the examined samples. The usage of highly sensitive assembler facilitated the discovery of a corneal transcriptome with an unprecedented length of $1.39 \mathrm{~Gb}$, compared with $289 \mathrm{Mb}$ in the reference GENCODE 21 transcriptome. Although some of the identified novel transcripts are likely assembly artifacts or cases of aberrant splicing, a study by Mercer et a ${ }^{30}$ showed that many of them can be biologically relevant; therefore, all detected transcripts were kept for further analysis. The expression values of the detected transcripts spanned many orders of magnitude, some as low as 0.001 TPM, well below the expression threshold for transcripts present in every cell of a given tissue. ${ }^{31}$

A further insight into the RNA-Seq results, particularly gene expression data, revealed considerable differences among the nonKTCN samples, some of which were more similar to the KTCN samples. This behavior can be attributed to the high heterogeneity of the control group used in this study, as it was not possible to obtain 'healthy' corneas from living individuals for comparative analyses. A probable explanation for the similarity in transcriptomes between the KTCN and non-KTCN samples is that certain pathologies of the cornea may share some molecular pathways with KTCN. The inclusion of KTCN and non-KTCN samples with some extent of overlapping transcriptomics data may be a confounding factor in this study. The relatively high number of removed samples in the discovery 
Table 1 Top pathways overrepresented across the differentially expressed genes detected by ConsensusPathDB server $(q$-value $<0.01)$

\begin{tabular}{|c|c|c|c|}
\hline Pathway name & Source & $q$-value & Genes \\
\hline \multicolumn{4}{|l|}{ Upregulated genes } \\
\hline CREB phosphorylation through the activation of Ras & Reactome & 0.00327180625385 & GRIN2A, GRIN2B, RPS6KA2, RPS6KA3, RPS6KA6 \\
\hline Post NMDA receptor activation events & Reactome & 0.00327180625385 & GRIN2A, GRIN2B, RPS6KA2, RPS6KA3, RPS6KA6 \\
\hline Neuronal system & Reactome & 0.00327180625385 & $\begin{array}{l}\text { CACNA1E, DLG3, GLUL, GRIN2A, GRIN2B, KCNG3, } \\
\text { KCNMB2, RPS6KA2, RPS6KA3, RPS6KA6 }\end{array}$ \\
\hline $\begin{array}{l}\text { Activation of NMDA receptor upon glutamate binding and } \\
\text { postsynaptic events }\end{array}$ & Reactome & 0.00327180625385 & GRIN2A, GRIN2B, RPS6KA2, RPS6KA3, RPS6KA6 \\
\hline Circadian entrainment - Homo sapiens (human) & KEGG & 0.00327180625385 & $\begin{array}{l}\text { CACNA1D, CACNA1H, CACNA1I, GRIN2A, GRIN2B, } \\
\text { NOS1AP, RPS6KA5 }\end{array}$ \\
\hline L1CAM interactions & Reactome & 0.00549551421988 & $\begin{array}{l}\text { DLG3, RPS6KA2, RPS6KA3, RPS6KA5, RPS6KA6, } \\
\text { SCN11A, SCN3A }\end{array}$ \\
\hline
\end{tabular}

Downregulated genes

Extracellular matrix organization

Reactome

$2.82499684672 \mathrm{e}-16$

Collagen formation

Collagen biosynthesis and modifying enzymes

Cytokine-cytokine receptor interaction - Homo sapiens (human) KEGG

Focal adhesion - Homo sapiens (human)

Hemostasis

ACTN1, ADAM9, ADAMTS1, BMP1, BMP4, CASK, CD47, CEACAM1, COL10A1, COL12A1, COL16A1, COL23A1, COL5A2, COL6A1, COL6A2, COL6A3, COL6A6, COL7A1, CTSB, CTSK, CTSS, DDR2, EFEMP1, EFEMP2, FBLN2, FBLN5, HSPG2, ICAM3, ITGA1, ITGA5, ITGB1, ITGB5, JAM3, LAMA1, LAMA2, LAMA4, LAMB3, LAMC1, LEPRE1, LEPREL1, LEPREL2, LOX, LOXL1, LOXL3, LTBP2, LUM, NID1, P4HB, PCOLCE, PLOD2, PLOD3, PPIB, SDC3, SERPINH1, SPARC, TGFB1, TGFB3, THBS1, TIMP1, TIMP2

Reactome

$5.2963761811 \mathrm{e}-05$

Reactome

Reactome

2.0777825037e-07

4.193887379e-06

$1.1201792372 \mathrm{e}-05$

KEGG 8.27597983794e-05
BMP1, COL10A1, COL12A1, COL16A1, COL23A1, COL5A2, COL6A1, COL6A2, COL6A3, COL6A6, COL7A1, CTSB, CTSS, LAMB3, LEPRE1, LEPREL1, LEPREL2, LOX, LOXL1, LOXL3, P4HB, PCOLCE, PLOD2, PLOD3, PPIB, SERPINH1 BMP1, COL10A1, COL12A1, COL16A1, COL23A1, COL5A2, COL6A1, COL6A2, COL6A3, COL6A6, COL7A1, LEPRE1, LEPREL1, LEPREL2, P4HB, PCOLCE, PLOD2, PLOD3, PPIB, SERPINH1 ACKR3, BMPR2, CSF1, CSF2RA, CX3CR1, CXCL16, EDA2R, FLT3LG, IFNGR2, IL13RA1, IL15, IL18R1, IL1R1, IL1RAP, IL22RA1, LIFR, OSMR, PDGFC, PDGFRA, PDGFRB, PLEKHO2, TGFB1, TGFB3, TGFBR1, TGFBR2, TNFRSF10B, TNFRSF11B, TNFRSF12A, TNFRSF21

ACTB, ACTN1, AKT3, BCL2, CAV1, CAV2, COL5A2, COL6A1, COL6A2, COL6A3, COL6A6, FLNA, FYN, ITGA1, ITGA5, ITGB1, ITGB5, JUN, LAMA1, LAMA2, LAMA4, LAMB3, LAMC1, MYL12A, MYL9, MYLK, PARVB, PDGFC, PDGFD, PDGFRA, PDGFRB, PIK3CG, PIK3R3, SHC1, THBS1, THBS2, VAV1, VCL ACTN1, AKT3, CALU, CAV1, CD47, CD63, CEACAM1, CENPE, CSF2RA, DOCK10, DOCK11, DOCK4, EHD2, EHD3, F2R, F3, FLNA, FYN, GAB2, GAS6, GNB4, HIST2H3A, HIST2H3C, INPP5D, ITGA1, ITGA5, ITGB1, JAK3, JAM3, KIF18A, KIF20A, KIF3C, LYN, MRVI1, P2RY1, PDE11A, PDE5A, PDPN, PIK3CG, PIK3R3, PLCG2, PRKACB, PROS1, S100A10, SERPINE2, SERPING1, SH2B3, SHC1, SIRPA, SLC16A1, SLC8A1, SPARC, SPN, TEK, TGFB1, TGFB3, THBS1, TIMP1, VAV1, VCL

ACTB, ADCY3, CACNA2D1, CACNA2D4, CACNB1, ITGA1, ITGA5, ITGB1, ITGB5, LAMA2, PRKACB, SGCB, SGCD, SLC8A1, TGFB1, TGFB3, TPM2, TPM4 
Table 1 (Continued)

\begin{tabular}{|c|c|c|c|}
\hline Pathway name & Source & q-value & Genes \\
\hline Non-integrin membrane-ECM interactions & Reactome & $9.55895386361 \mathrm{e}-05$ & $\begin{array}{l}\text { ACTN1, CASK, DDR2, HSPG2, ITGB1, ITGB5, LAMA1, } \\
\text { LAMA2, LAMA4, LAMB3, LAMC1, SDC3, TGFB1, } \\
\text { THBS1 }\end{array}$ \\
\hline Signal transduction & Reactome & 0.000134135175937 & $\begin{array}{l}\text { ACKR3, ACTB, ADAM12, ADCY3, AKT3, AMOT, ANXA1, } \\
\text { ARHGAP11A, ARHGAP20, ARHGAP24, ARHGAP26, } \\
\text { ARHGAP31, ARHGEF25, AXIN2, AXL, B4GALT1, } \\
\text { BAMBI, BMPR2, BUB1, CAMK2D, CAV1, CCDC88C, } \\
\text { CD86, CDC42EP3, CDC42EP5, CDH5, CDKN1A, } \\
\text { CENPE, CENPF, CHN1, CLIP1, COL6A1, COL6A2, } \\
\text { COL6A3, COL6A6, CRABP2, CSF2RA, CX3CR1, } \\
\text { CXCL16, CYP26B1, DIAPH3, DLC1, DLG4, DNM1, } \\
\text { DTX4, DUSP10, DUSP4, DZIP1, E2F3, EDNRB, ESR1, } \\
\text { F2R, FGD2, FGFR1, FLNA, FMNL2, FMNL3, FSTL1, } \\
\text { FYN, FZD1, FZD7, GAB2, GLI2, GLI3, GNB4, GPR161, } \\
\text { GPR37, GRB10, GRP, HDAC9, HHIP, HIF1A, } \\
\text { HIST1H2AB, HIST1H2BH, HIST1H2BJ, HIST1H2BK, } \\
\text { HIST1H2BN, HIST2H3A, HIST2H3C, HIST2H4B, } \\
\text { HMHA1, HRH1, HSPG2, IL17RD, IQGAP2, IRS1, IRS2, } \\
\text { ITGB1, JAK3, JUN, KIF18A, KIF7, LATS2, LIMK1, LYN, } \\
\text { MYL9, MYLK, NCF4, NEURL1B, NFATC1, NPBWR1, } \\
\text { NRP1, OPN3, P2RY1, P4HB, PDE11A, PDE4B, PDGFC, } \\
\text { PDGFD, PDGFRA, PDGFRB, PIK3CG, PIK3R3, PLB1, } \\
\text { PLEKHG2, PPP1R15A, PRICKLE1, PRKACB, PTGER4, } \\
\text { PYG01, RASGRF2, RGS19, ROR2, RTN4, S1PR2, } \\
\text { SAA1, SDC3, SFN, SH2B3, SHC1, SKIL, SMAD6, } \\
\text { SMAD7, SM0, SOX4, SOX9, SPC25, SPTBN1, } \\
\text { STARD13, STARD8, STAT3, SYDE1, TCF4, TEK, } \\
\text { TGFB1, TGFBR1, TGFBR2, THBS1, THBS2, TLE4, } \\
\text { TNFAIP3, TNFRSF10B, TRIO, VANGL2, VAV1, VCL, } \\
\text { WIPF1, WNT5A, YWHAH }\end{array}$ \\
\hline Assembly of collagen fibrils and other multimeric structures & Reactome & 0.000159161289426 & $\begin{array}{l}\text { BMP1, COL10A1, COL6A1, COL6A2, COL6A3, } \\
\text { COL6A6, COL7A1, CTSB, CTSS, LAMB3, LOX, LOXL1, } \\
\text { LOXL3, PCOLCE }\end{array}$ \\
\hline Hippo signaling pathway - Homo sapiens (human) & KEGG & 0.000278732976836 & $\begin{array}{l}\text { ACTB, AMOT, AREG, AXIN2, BBC3, BMP4, BMPR2, } \\
\text { CTGF, DLG4, FZD1, FZD7, GLI2, ID2, LATS2, NKD1, } \\
\text { PPP2R2B, SMAD7, SNAI2, TEAD2, TEAD4, TGFB1, } \\
\text { TGFB3, TGFBR1, TGFBR2, WNT5A, WTIP, YWHAH }\end{array}$ \\
\hline Hypertrophic cardiomyopathy (HCM) - Homo sapiens (human) & KEGG & 0.000333844038681 & $\begin{array}{l}\text { ACTB, CACNA2D1, CACNA2D4, CACNB1, ITGA1, } \\
\text { ITGA5, ITGB1, ITGB5, LAMA2, SGCB, SGCD, SLC8A1, } \\
\text { TGFB1, TGFB3, TPM2, TPM4 }\end{array}$ \\
\hline Proteoglycans in cancer - Homo sapiens (human) & KEGG & 0.000333844038681 & $\begin{array}{l}\text { ACTB, AKT3, ANK3, CAMK2D, CAV1, CAV2, CD63, } \\
\text { CDKN1A, ESR1, FGFR1, FLNA, FZD1, FZD7, HIF1A, } \\
\text { HSPG2, ITGA5, ITGB1, ITGB5, LUM, MRAS, MSN, } \\
\text { PIK3CG, PIK3R3, PLCG2, PRKACB, SMO, STAT3, } \\
\text { TGFB1, THBS1, TIMP3, TWIST1, TWIST2, WNT5A }\end{array}$ \\
\hline ECM-receptor interaction - Homo sapiens (human) & KEGG & 0.000419450934734 & $\begin{array}{l}\text { CD47, COL5A2, COL6A1, COL6A2, COL6A3, COL6A6, } \\
\text { HSPG2, ITGA1, ITGA5, ITGB1, ITGB5, LAMA1, LAMA2, } \\
\text { LAMA4, LAMB3, LAMC1, THBS1, THBS2 }\end{array}$ \\
\hline Elastic fiber formation & Reactome & 0.000438089241869 & $\begin{array}{l}\text { BMP4, EFEMP1, EFEMP2, FBLN2, FBLN5, ITGA5, } \\
\text { ITGB1, ITGB5, LTBP2, TGFB1, TGFB3 }\end{array}$ \\
\hline
\end{tabular}

study (3 of 8 non-KTCN samples) is likely the effect of random sampling, as only 2 such samples were observed among the 17 nonKTCN samples in the replication experiment.

Comparisons of the differential expression results of the discovery and replication studies showed that $82.55 \%$ of genes and $74.96 \%$ of transcripts detected as differentially expressed during the discovery study and having enough coverage to be included in both studies were also found in the replication experiment. These confirmed results prove that the methodology used in this study can provide reproducible results, which is particularly important given the very low reproducibility rate of published results in the medical sciences. ${ }^{32,33}$ This result was achieved mainly by using recently developed, state-ofthe-art programs for RNA-Seq data analysis and combining them into a pipeline in a form not previously used. Moreover, results of RT- 
Table 2 Genes involved in collagen synthesis and cornea structure formation altered in KTCN corneas

\begin{tabular}{|c|c|c|c|c|c|c|c|}
\hline \multirow[b]{2}{*}{ Collagen genes } & \multirow[b]{2}{*}{ Hydroxylation } & \multicolumn{2}{|c|}{ Collagen synthesis } & \multirow[b]{2}{*}{ Proteolytic conversion } & \multirow[b]{2}{*}{ Lys oxydation } & \multicolumn{2}{|c|}{ Structure of the cornea } \\
\hline & & Glycosilation & Formation of triple helix & & & Proteoglycans & Corneal structure \\
\hline$\downarrow C O L 5 A 2$ & $\downarrow P 4 H A 2$ & $\downarrow P L O D 3$ & $\downarrow P P I B$ & $\downarrow$ ADAMTS1 & $\downarrow L O X$ & $\downarrow L U M$ & $\downarrow L A M A 1$ \\
\hline$\downarrow$ COL6A2 & $\downarrow P L O D 2$ & & & $\downarrow$ ADAMTS10 & $\downarrow L O X L 3$ & & $\downarrow L A M A 4$ \\
\hline$\downarrow C O L 6 A 3$ & $\downarrow P L O D 3$ & & & $\downarrow A D A M 9$ & & & $\downarrow \angle A M B 3$ \\
\hline$\downarrow$ COLGAG & & & & $\downarrow A D A M 12$ & & & $\downarrow \angle A M C 1$ \\
\hline$\downarrow$ COL12A1 & & & & $\uparrow A D A M T S 17$ & & & \\
\hline \multicolumn{8}{|l|}{$\downarrow$ COL16A1 } \\
\hline \multicolumn{8}{|l|}{$\downarrow$ COL23A1 } \\
\hline \multicolumn{8}{|l|}{$\uparrow \operatorname{COL} 21 A 1$} \\
\hline$\uparrow \operatorname{COL28A1}$ & & & & & & & \\
\hline
\end{tabular}

$\downarrow$ Indicates downregulated genes.

$\uparrow$ Indicates upregulated genes.

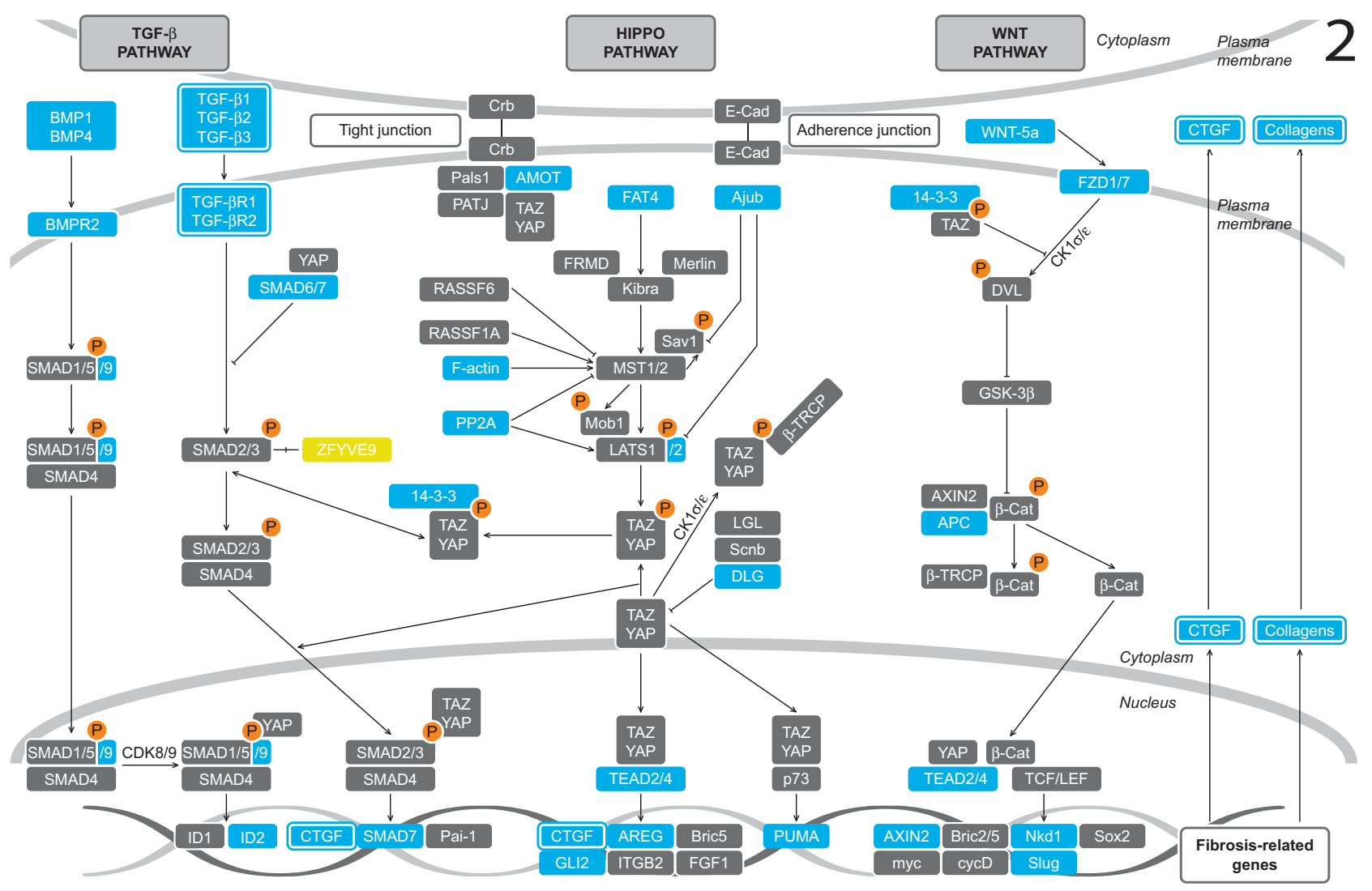

Legend:
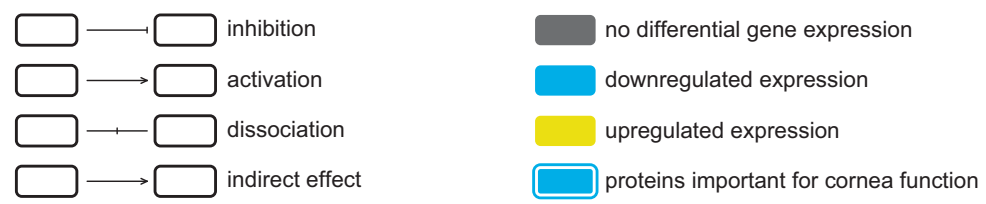

P phosphorylation

Figure 3 Dysregulated TGF- $\beta$, Hippo, and Wnt pathways in KTCN corneas. Yellow rectangle indicates gene with upregulated expression in KTCN corneas; genes with downregulated expression are shown in blue rectangles. Genes without detectable expression alterations but that are core elements of the pathways are also included and shown in gray rectangles. Downregulated genes, important for functioning of the cornea, are shown as rectangles with additional frames. Statistically significant downregulation of gene expression in KTCN corneas is visible in the presented pathways, whereas only one upregulated gene is exposed. 
qPCR analyses of selected genes performed in KTCN and non-KTCN individuals were consistent with RNA-Seq data, providing independent confirmation of RNA-Seq data.

The pathway overrepresentation analysis showed that only six pathways from the KEGG and Reactome databases were statistically significantly overrepresented in the upregulated gene set, including genes involved in the functioning of the neuronal system. Of those, GRIN2A, GRIN2B, RPS6KA2, RPS6KA3, and RPS6KA6 have a role in establishing long-lasting synaptic changes or initiating the signaling cascade involved in the process of learning. Thus, we suggest that there is no clear link between dysregulation of these particular enriched molecular pathways and the characteristic changes observed in KTCN corneas. However, taking into account that the current state of knowledge regarding KTCN biology is incomplete, their connection to KTCN cannot be fully excluded. Downregulated genes, on the other hand, produced results that were much more informative, showing significant dysregulation of 51 pathways in KTCN, including collagen synthesis, ECM formation, and cytokine signaling.

Interestingly, in this study, a significant increase was observed in the expression of COL21A1, whereas significant decrease was observed in the expression of other collagens, including COL5A2, COL6A1, COL6A2, COL6A3, COL6A6, and COL12A1, which encode the major structural components of the human cornea. In a previous study, a mass spectrometry assessment of protein levels in the human corneal epithelium and stroma showed that all but one of these collagens, COL5A2, had decreased. ${ }^{34}$ Thus, we suppose that in these genes, dysregulation of their expression may have a direct impact on protein levels.

In contrast to the non-KTCN samples, lower expression of nearly all genes involved in collagen maturation was observed in the KTCN samples, including the LOX gene, whose inhibition reduces the formation of cross-links in collagen and decreases the mechanical stability of the matrix. ${ }^{35}$ The current results are in line with previous findings of decreased LOX expression in the corneal epithelium of human KTCN corneas as measured by $\mathrm{qPCR}^{36} \mathrm{We}$ propose that the finding of extensive disruption of the collagen synthesis pathway, resulting in the decreased expression of collagens and collagen-related genes in KTCN corneas, is not incidental, as it is known that thinning of a KTCN cornea may be the final effect of a disorganized collagen lamellae arrangement. The latest ultrastructural analysis of keratoconic corneal buttons, using transmission electron microscopy, suggested that KTCN is associated with the splitting of existing collagen lamellae into smaller units, which results in a higher total lamellar count. ${ }^{37}$ The mechanism of lamellar alteration in KTCN stroma is not fully understood. However, several studies have indicated that collagen slippage observed in KTCN corneas may be due to a loss of cohesion between collagen fibrils and other ECM components. ${ }^{38}$

In addition to collagens, other ECM molecules, including proteoglycans, have a role in fibrillogenesis and matrix assembly in human corneas. ${ }^{39}$ Recently, it was shown that one of the major corneal proteoglycans, $L U M$ - encoding lumican, was downregulated in cell cultures derived from KTCN corneas and in the corneas. ${ }^{34,40}$ In this study, the expression of LUM, was also diminished, which could destabilize normal corneal fibril organization.

The ECM is secreted and assembled by keratocytes in a process regulated by the cytokine TGF- $\beta$. The three TGF- $\beta$ isoforms, TGFB1, TGFB2, and TGFB3, whose secretion and binding initiate signaling, are known to be important modulators of ECM remodeling. TGFB1 and TGFB2 are recognized as stimulators of a profibrotic response following injury, whereas TGFB3 is known for its antifibrotic effects, resulting in the production of normal ECM components. ${ }^{41}$ In the injured cornea, TGFB1 binds to TGF receptor II (TGFBR2) and stimulates the synthesis of CTGF, which activates keratocytes directly to transform into activated cells that produce collagen. In this study, the analysis showed a statistically significant decrease in the expression of two TGF- $\beta$ ligands encoded by TGFB1 and TGFB3 genes, their receptors, encoded by TGFBR1 and TGFBR2, and CTGF in keratoconic corneas. This finding suggests that low expression of genes encoding major ECM components, including collagens, may be an effect of TGF- $\beta$ signaling pathway alteration. In a previous study, it was shown that stimulation of human KTCN cells by TGF- $\beta$ leads to the generation of ECM components in amounts similar to those generated by normal corneal fibroblasts. ${ }^{42}$ However, although CTGF expression in fibroblasts is considered to be induced primarily by genes encoding TGF- $\beta$ ligands, ${ }^{43}$ two other signaling pathways, Hippo and Wnt, can directly and indirectly regulate TGF- $\beta$ pathway activity and the production of CTGF protein. ${ }^{25}$ These three pathways are integrated by YAP and TAZ molecules positioned in their center. It has been hypothesized that signaling crosstalk among elements of the TGF- $\beta$, Hippo, and Wnt pathways may have a role in ocular health. ${ }^{44}$

We observed in this study that the core elements of the TGF- $\beta$ pathway (genes indicated above), the Hippo pathway (FAT4, LATS2, TAED2, TAED4), and the Wnt pathway (WNT5A, FZD1, FZD7) were downregulated in the KTCN samples. Although the dysregulation of genes coding several elements of the TGF- $\beta$ pathway has previously been reported in KTCN samples, ${ }^{41,45}$ changes in the Hippo and Wnt pathways have not been reported in KTCN. However, because it is known that Wnt signaling has a significant role in regulating the proliferation of corneal epithelial stem cells, and because the Hippo pathway is widely recognized as a signaling pathway that regulates organ size, including eyes, ${ }^{46,47}$ we suggest that the dysregulation of genes involved in these pathways is not incidental in KTCN. Taken together, our results confirm the hypothesis that KTCN may be linked to altered TGF- $\beta$ signaling ${ }^{41}$ and indicate two other pathways not previously mentioned that are dysregulated in KTCN corneas. However, the fact that the TGF- $\beta$, Hippo, and Wnt pathways are intertwined makes it difficult to determine which of their functional elements may be the original factor responsible for the etiology of the disease. The exact cause of the downregulation of the TGF- $\beta$, Hippo, and Wnt pathways also remains elusive.

In summary, despite a great degree of genetic heterogeneity in KTCN, common downstream mechanisms, including gene expression, may be altered, as proven in this report. We found significant alterations in the transcriptome profile of KTCN compared with non-KTCN corneas, manifested particularly in collagen synthesis disruption and downregulation of core elements of the TGF- $\beta$, Hippo, and Wnt pathways. The important aspects are that the same alterations were shared by all affected individuals and that these results were confirmed in the following replication study. This study is the first report to show the extent and complexity of corneal KTCN transcriptomes. Together, observing the disruption of collagen synthesis, the TGF- $\beta$, Hippo, and Wnt pathways, bring new insights into the etiology of KTCN and the role of common signaling pathways in the disease.

\section{Availability of data and materials}

Sequencing data are available in the Gene Expression Omnibus database (GSE77938).

\section{CONFLICT OF INTEREST}

The authors declare no conflict of interest. 


\section{ACKNOWLEDGEMENTS}

This study was supported by National Science Centre in Poland, Grant 2012/05/E/NZ5/02127 (to MG).

1 Rabinowitz YS: Keratoconus. Surv Ophthalmol 1998; 42: 297-319.

2 Dobbins KR, Price FW, Whitson WE: Trends in the indications for penetrating keratoplasty in the midwestern United States. Cornea 2000; 19: 813-816.

3 Abu-Amero KK, Al-Muammar AM, Kondkar AA: Genetics of keratoconus: where do we stand? J Ophthalmol 2014; 2014: 641708.

4 Galvis V, Sherwin T, Tello A, Merayo J, Barrera R, Acera A: Keratoconus: an inflammatory disorder? Eye Lond Engl 2015; 29: 843-859.

5 Héon E, Greenberg A, Kopp KK et al: VSX1: a gene for posterior polymorphous dystrophy and keratoconus. Hum Mol Genet 2002; 11: 1029-1036.

6 Burdon KP, Macgregor S, Bykhovskaya Y et al: Association of polymorphisms in the hepatocyte growth factor gene promoter with keratoconus. Invest Ophthalmol Vis Sci 2011; 52: 8514-8519.

7 Hughes AE, Bradley DT, Campbell M et al: Mutation altering the miR-184 seed region causes familial keratoconus with cataract. Am J Hum Genet 2011; 89: 628-633.

8 Gajecka M, Radhakrishna U, Winters D et al: Localization of a gene for keratoconus to a 5.6-Mb interval on 13q32. Invest Ophthalmol Vis Sci 2009; 50: 1531-1539.

9 Karolak JA, Kulinska K, Nowak DM et al: Sequence variants in COL4A1 and COL4A2 genes in Ecuadorian families with keratoconus. Mol Vis 2011; 17: 827-843.

10 Czugala M, Karolak JA, Nowak DM et al: Novel mutation and three other sequence variants segregating with phenotype at keratoconus $13 q 32$ susceptibility locus. Eur J Hum Genet 2012; 20: 389-397.

11 Karolak JA, Rydzanicz M, Ginter-Matuszewska B et al: Variant c.2262A >C in DoCK9 leads to exon skipping in keratoconus family. Invest Ophthalmol Vis Sci 2015; 56: 7687-7690.

12 Karolak JA, Polakowski P, Szaflik J, Szaflik JP, Gajecka M: Molecular screening of keratoconus susceptibility sequence variants in VSX1, TGFBI, DOCK9, STK24, and IPO5 genes in Polish patients and novel TGFBI variant identification. Ophthalmic Genet 2016; 37: 37-43.

13 Nielsen K, Heegaard S, Vorum H, Birkenkamp-Demtröder K, Ehlers N, Orntoft TF: Altered expression of CLC, DSG3, EMP3, S100A2, and SLPI in corneal epithelium from keratoconus patients. Cornea 2005; 24: 661-668.

14 Macé M, Galiacy SD, Erraud A et al: Comparative transcriptome and network biology analyses demonstrate antiproliferative and hyperapoptotic phenotypes in human keratoconus corneas. Invest Ophthalmol Vis Sci 2011; 52: 6181-6191.

15 Bolger AM, Lohse M, Usadel B: Trimmomatic: a flexible trimmer for Illumina sequence data. Bioinforma Oxf Eng/ 2014; 30: 2114-2120.

16 Langmead B, Salzberg SL: Fast gapped-read alignment with Bowtie 2. Nat Methods 2012; 9: 357-359.

17 Dobin A, Davis CA, Schlesinger F et al: STAR: ultrafast universal RNA-seq aligner. Bioinforma Oxf Engl 2013; 29: 15-21.

18 Pertea M, Pertea GM, Antonescu CM, Chang T-C, Mendell JT, Salzberg SL: StringTie enables improved reconstruction of a transcriptome from RNA-seq reads. Nat Biotechnol 2015; 33: 290-295.

19 Roberts A, Pimentel H, Trapnell C, Pachter L: Identification of novel transcripts in annotated genomes using RNA-Seq. Bioinforma Oxf Engl 2011; 27: 2325-2329.

20 Haas BJ, Papanicolaou A, Yassour M et al: De novo transcript sequence reconstruction from RNA-seq using the Trinity platform for reference generation and analysis. Nat Protoc 2013; 8: 1494-1512.

21 Bray N, Pimentel H, Melsted P, Pachter L: Near-optimal RNA-Seq quantification. ArXiv150502710 Cs Q-Bio 2015, http://arxiv.org/abs/1505.02710 (accessed 31 March 2016)

22 Love MI, Huber W, Anders S: Moderated estimation of fold change and dispersion for RNA-seq data with DESeq2. Genome Biol 2014; 15: 550.
23 Robinson MD, McCarthy DJ, Smyth GK: edgeR: a Bioconductor package for differential expression analysis of digital gene expression data. Bioinforma Oxf Engl 2010; 26: 139-140.

24 Kamburov A, Stelzl U, Lehrach H, Herwig R: The ConsensusPathDB interaction database: 2013 update. Nucleic Acids Res 2013; 41: D793-D800.

25 Attisano L, Wrana JL: Signal integration in TGF- $\beta$, WNT, and Hippo pathways. F1000prime Rep 2013; 5: 17.

26 Liu Y, Zhou J, White KP: RNA-seq differential expression studies: more sequence or more replication? Bioinforma Oxf Eng/ 2014; 30: 301-304.

27 Ha NT, Nakayasu K, Murakami A, Ishidoh K, Kanai A: Microarray analysis identified differentially expressed genes in keratocytes from keratoconus patients. Curr Eye Res 2004; 28: 373-379.

28 Nielsen K, Birkenkamp-Demtröder K, Ehlers N, Orntoft TF: Identification of differentially expressed genes in keratoconus epithelium analyzed on microarrays. Invest Ophthalmol Vis Sci 2003; 44: 2466-2476.

29 Joseph R, Srivastava OP, Pfister RR: Modeling keratoconus using induced pluripotent stem cells. Invest Ophthalmol Vis Sci 2016; 57: 3685-3697.

30 Mercer TR, Gerhardt DJ, Dinger ME et al: Targeted RNA sequencing reveals the deep complexity of the human transcriptome. Nat Biotechnol 2012; 30: 99-104.

31 Roberts A, Pachter L: RNA-Seq and find: entering the RNA deep field. Genome Med 2011; 3: 74.

32 Prinz F, Schlange T, Asadullah K: Believe it or not: how much can we rely on published data on potential drug targets? Nat Rev Drug Discov 2011; 10: 712.

33 Begley CG, Ellis LM: Drug development: raise standards for preclinical cancer research. Nature 2012; 483: 531-533.

34 Chaerkady R, Shao H, Scott S-G, Pandey A, Jun AS, Chakravarti S: The keratoconus corneal proteome: loss of epithelial integrity and stromal degeneration. J Proteomics 2013; 87: 122-131.

35 Parapuram SK, Hodge W: The integrin needle in the stromal haystack: emerging role in corneal physiology and pathology. J Cell Commun Signal 2014; 8: 113-124.

36 Shetty R, Sathyanarayanamoorthy A, Ramachandra RA et al: Attenuation of lysyl oxidase and collagen gene expression in keratoconus patient corneal epithelium corresponds to disease severity. Mol Vis 2015; 21: 12-25.

37 Mathew JH, Goosey JD, Söderberg PG, Bergmanson JPG: Lamellar changes in the keratoconic cornea. Acta Ophthalmol (Copenh) 2015; 93: 767-773.

38 Meek KM, Tuft SJ, Huang Y et al: Changes in collagen orientation and distribution in keratoconus corneas. Invest Ophthalmol Vis Sci 2005; 46: 1948-1956.

39 Massoudi D, Malecaze F, Galiacy SD: Collagens and proteoglycans of the cornea: importance in transparency and visual disorders. Cell Tissue Res 2016; 363: 337-349.

40 García B, García-Suárez 0, Merayo-Lloves J et al: Differential expression of proteoglycans by corneal stromal cells in keratoconus. Invest Ophthalmol Vis Sci 2016; 57: 2618-2628.

41 Priyadarsini S, McKay TB, Sarker-Nag A, Karamichos D: Keratoconus in vitro and the key players of the TGF- $\beta$ pathway. Mol Vis 2015; 21: 577-588.

42 Lyon D', McKay TB, Sarkar-Nag A, Priyadarsini S, Karamichos D: Human keratoconus cell contractility is mediated by transforming growth factor-beta isoforms. J Funct Biomater 2015; 6: 422-438.

43 Grotendorst GR: Connective tissue growth factor: a mediator of TGF-beta action on fibroblasts. Cytokine Growth Factor Rev 1997; 8: 171-179.

44 Morgan JT, Murphy CJ, Russell P: What do mechanotransduction, Hippo, Wnt, and TGF $\beta$ have in common? YAP and TAZ as key orchestrating molecules in ocular health and disease. Exp Eye Res 2013; 115: 1-12.

45 Engler C, Chakravarti S, Doyle J et al: Transforming growth factor- $\beta$ signaling pathway activation in Keratoconus. Am J Ophthalmol 2011; 151: 752-759,e2.

46 Nakatsu MN, Ding Z, Ng MY, Truong TT, Yu F, Deng SX: Wnt/ $\beta$-catenin signaling regulates proliferation of human cornea epithelial stem/progenitor cells. Invest Ophthalmol Vis Sci 2011; 52: 4734-4741.

47 Yu F-X, Guan K-L: The Hippo pathway: regulators and regulations. Genes Dev 2013; 27: 355-371.

Supplementary Information accompanies this paper on European Journal of Human Genetics website (http://www.nature.com/ejhg) 